\title{
Synthesis and Structure of Ferrocenol Esters
}

\author{
Mikhail Denisov1, Aleksey Gorbunov, Maksim V. Dmitriev², Pavel Slepukhin ${ }^{3,4}$, \\ Vladimir Glushkov ${ }^{1,2}$ \\ ${ }^{1}$ Institute of Technical Chemistry, Urals Branch of the Russian Academy of Sciences, Perm, Russia \\ ${ }^{2}$ Perm State National Research University, Perm, Russia \\ ${ }^{3}$ I. Ya. Postovsky Institute of Organic Synthesis, Urals Branch of the Russian Academy of Sciences, Ekaterinburg, \\ Russia \\ ${ }^{4}$ Ural Federal University, Ekaterinburg, Russia \\ Email: glusha55@gmail.com
}

Received 4 February 2016; accepted 28 May 2016; published 31 May 2016

Copyright $(\subset 2016$ by authors and Scientific Research Publishing Inc.

This work is licensed under the Creative Commons Attribution International License (CC BY).

http://creativecommons.org/licenses/by/4.0/

(c) (i) Open Access

\begin{abstract}
$\mathrm{N}$-Heterocyclic carbene-catalyzed oxidative esterification of ferroceneboronic acid by aromatic and heteroaromatic aldehydes affords the new ferrocenol hetaryl and aryl esters 1 - 4, 6 - 8 and 9 11. The reaction takes place under mild conditions. The $X$-ray crystal structure of ferrocenyl esters $3,6,11$ was determined.
\end{abstract}

\section{Keywords}

Hydroxyferrocene, Ferroceneboronic Acid, N-Heterocyclic Carbene, Esterification, Catalysis

\section{Introduction}

Ferrocene is considered to be one of the most prominent molecules in modern organic and organometallic chemistry [1]-[3]. Recently, ferrocenyl-substituted compounds have found widespread application in medicinal chemistry [4]-[16], material science [17]-[21] and asymmetric catalysis [22]-[25]. Ferrocene is a source for artificial receptors [26], biosensors [27]-[29], liquid crystals [30] [31] and redox-active structures [32]-[37].

Nesmeyanov first reported hydroxyferrocene in 1959, generating it from either ferroceneboronic acid FcB $(\mathrm{OH})_{2}$ (via reaction with $\mathrm{Cu}(\mathrm{OAc})_{2}$ and then potassium hydroxide) [38] or by alkaline hydrolysis of the acetate generated from $\mathrm{FcBr}$ and $\mathrm{Cu}(\mathrm{OAc})_{2}$ [39]. Hydroxyferrocene is a yellow, very air-sensitive labile solid and a slightly weaker acid than phenol [40]. Due to relative instability its chemistry is not fully developed yet. Ferrocenol esters were synthesized by acylation of ferrocenol with carboxylic acid chlorides [41], but the more convenient method for their preparation is Cu-assisted reaction of haloferrocenes with carboxylic acids [39] [42][44]. Here we present a preparative route to ferrocenol esters from easily available ferroceneboronic acid [45]-

How to cite this paper: Denisov, M., Gorbunov, A., Dmitriev, M.V., Slepukhin, P. and Glushkov, V. (2016) Synthesis and Structure of Ferrocenol Esters. International Journal of Organic Chemistry, 6, 107-116. 
[47], avoiding isolation of hydroxyferrocene. The structure of esters deduced from their X-ray diffraction analysis is also discussed. Optical properties and electrochemical behavior of ferrocenol esters were reported in our preliminary communication [48].

\section{Results and Discussion}

\subsection{Synthesis}

Recently oxidative esterification of aromatic (heteroaromatic) aldehydes was disclosed [49]-[54]. The reaction utilizes an organocatalytic N-heterocyclic carbene activation-airobic oxidation of aldehydes in the presence of areneboronic acids. Involving $\mathrm{FcB}(\mathrm{OH})_{2}$ in this process gives straightforward route to ferrocenol esters (Scheme 1). Isolated yields of the compounds 1-11 varied within 16\% - 77\% (Table 1). The proposed reaction mechanism is discussed in literature [52] [53]. Carbene (1,3-dimesithylimidazole-2-ilidene) was generated in situ by the interaction of 1,3-dimethylimidazolidine chloride [55] with $\mathrm{Cs}_{2} \mathrm{CO}_{3}$.

Oxygen of the air is evidently the terminate oxidant. Therefore, we tried to bubble the air into the reaction vessel to improve the yield. However, only in the case of compound 11, the yield increased to some extent, with other compound yields being unaffected by this improvement.

At room temperature, esters 8 and 9 decompose during storage over 3 - 4 days. Indeed, the stability of esters 1-11 has a decisive influence on the yields (Table 1).

\subsection{The Structure of Ferrocenol Esters}

Below is shown the X-ray crystal structure of compound 1 (Figure 1), compound 4 (Figure 2) and compound 10 (Figure 3).

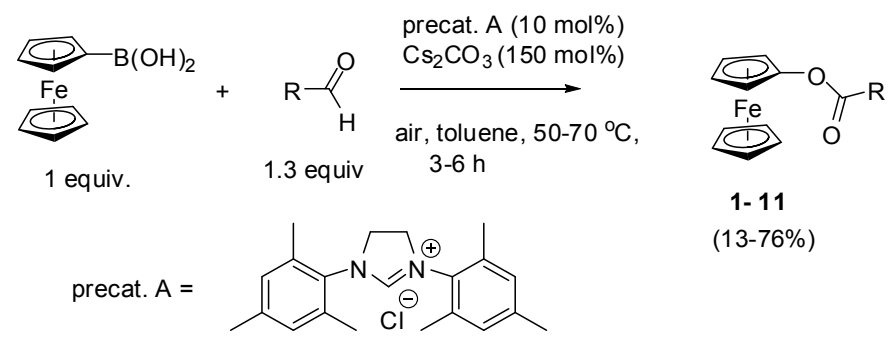

Scheme 1. Synthesis of compounds 1-11.

Table 1. Yields of compounds 1-11 and the reaction time.

\begin{tabular}{cccccc}
\hline Compound & $\mathrm{R}$ & Temperature, ${ }^{\circ} \mathrm{C}$ & Time $(\mathrm{h})$ & Flash mixture, petroleum ether-ethylacetate & Yield (\%) \\
\hline 1 & $3,4-(\mathrm{MeO})_{2} \mathrm{C}_{6} \mathrm{H}_{3}$ & 50 & 3 & $5: 1$ & 76 \\
2 & $3,4,5-(\mathrm{MeO})_{3} \mathrm{C}_{6} \mathrm{H}_{2}$ & 70 & 6 & $10: 1$ & 16 \\
3 & $4-(\mathrm{CF} 3) \mathrm{C}_{6} \mathrm{H}_{4}$ & 70 & 3 & $20: 1$ & 35 \\
4 & 1 -naphtyl & 50 & 6 & $10: 1$ & 76 \\
5 & $\mathrm{CH}=\mathrm{CHPh}$ & 50 & 3 & $10: 1$ & 36 \\
6 & 2 -furyl & 50 & 3 & $10: 1$ & 34 \\
7 & 2 -thienyl & 50 & 3 & $10: 1$ & 35 \\
8 & 2 -pyridyl & 50 & 6 & $5: 1$ & 17 \\
9 & 3-pyridyl & 50 & 6 & $3: 1$ & 33 \\
10 & 4-pyridyl & 50 & 6 & $3: 1$ & 17 \\
11 & ferrocenyl & 70 & 6 & $10: 1$ & 6 \\
$11^{\text {a }}$ & ferrocenyl & 70 & 6 & $10: 1$ & 28 \\
\hline
\end{tabular}

${ }^{\mathrm{a}}$ The air was bubbled into the reaction mixture. 


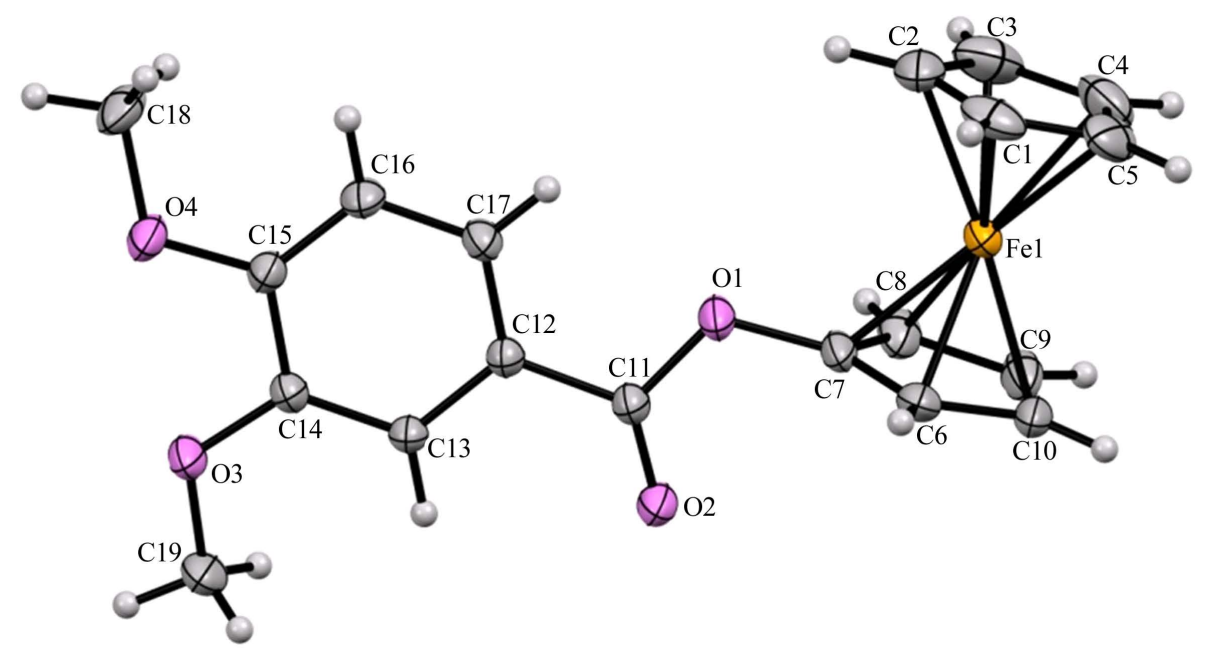

Figure 1. The X-ray structure of compound 1.

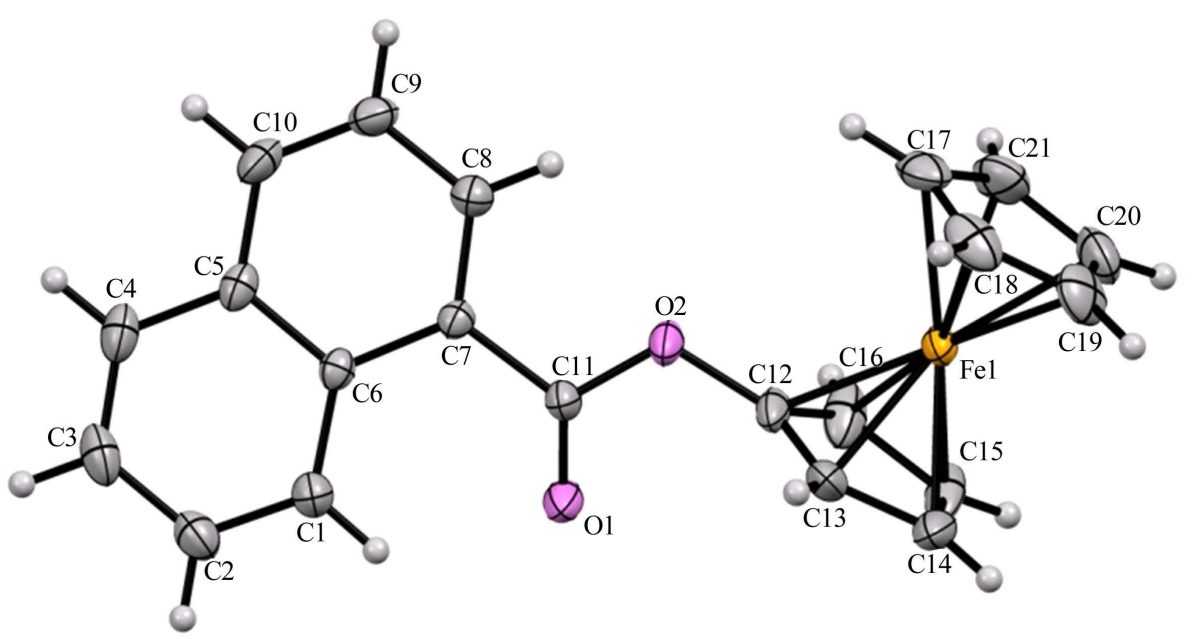

Figure 2. The X-ray structure of compound 4.

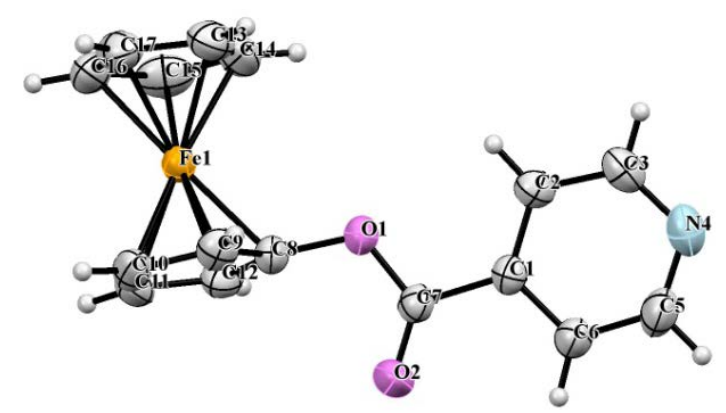

Figure 3. Compound $\mathbf{1 0}$ in the thermal ellipsoids of the $50 \%$ probability (one independent molecule).

In accord with XRD data, two independent molecules of ferrocenyl isonicotinate $\mathbf{1 0}$ are crystallized in the unit cell (Figure 4). The plane of the pyridine ring in molecules is approximately perpendicular towards the plane of the cyclopentadienyl ring; planes of the independent molecules in the unit cell are also approximately perpendicular. These structural features are similar to esters 1 and 4. No any deviations from standard bonds lengths or angles in the molecules are observed and no any shortened contacts are presented in the molecular packing. 


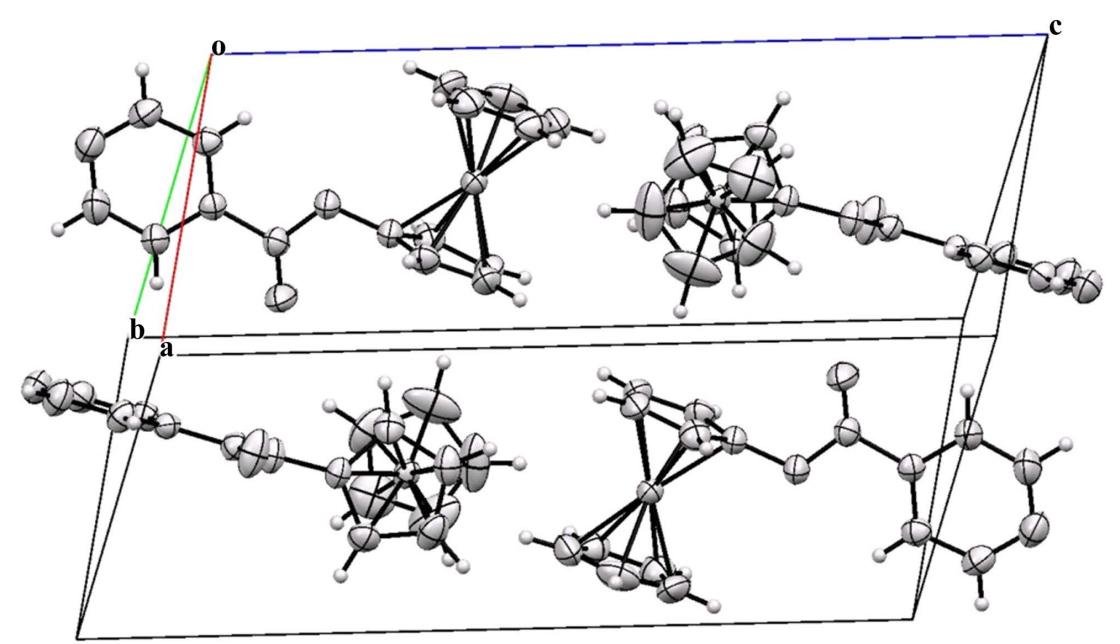

Figure 4. Two independent molecules in the unit cell of the compound $\mathbf{1 0 .}$

\section{Experimental}

\subsection{General}

The melting points were determined on the PTP apparatus and are uncorrected. NMR spectra were recorded in $\mathrm{CDCl}_{3}$ using a Varian Mercury Plus 300 at $300 \mathrm{MHz}\left({ }^{1} \mathrm{H}\right)$ and $75\left({ }^{13} \mathrm{C}\right) \mathrm{MHz}$. Chemical shifts were referenced to solvent signals $\left({ }^{13} \mathrm{C}\right)$ and GMDS $\left({ }^{1} \mathrm{H}\right)$. The IR spectra were recorded in Nujol on Bruker IFS 66 ps. Elemental analysis was carried out on CHNS Leco 9321P analyzer. The reaction mixture was qualitatively analyzed by GC-MS Agilent Technologies $6890 \mathrm{~N} / 5975 \mathrm{~B}$ system with HP-5 ms, 30,000 $\times 0.25 \mathrm{~mm}$ column. The column was heated up to $260^{\circ} \mathrm{C}$. The same device was used for recording mass-spectra (EI, $70 \mathrm{eV}$ ). The crude product was purified by column chromatography on Silica gel 60 (AlfaAesar, $0.032-0.070 \mathrm{~mm}$ ).

\subsection{General Method of Oxidative Esterification}

A mixture of ferroceneboronic acid $(0.105 \mathrm{~g}, 0.5 \mathrm{mmol})$, corresponding aldehyde $(0.65 \mathrm{mmol}), \mathrm{CsCO}_{3}(0.244 \mathrm{~g}$, $0.75 \mathrm{mmol})$ and 1,3-bis(2,4,6-trimethyphenyl)-4,5-dihydro- $1 \mathrm{H}$-imidazol-3-ium chloride $(0.017 \mathrm{~g}, 0.05 \mathrm{mmol})$ was suspended in toluene $(10 \mathrm{ml})$, and stirred in air at $50^{\circ} \mathrm{C}$ or at $70^{\circ} \mathrm{C}$ for 3 or $6 \mathrm{~h}$ (Table 1$)$. The solvent was removed under a vacuum, products were purified by flash chromatography on silica gel (petroleum ether- ethyl acetate), from 10:1 to 3:1 (Table 1).

\subsection{Ferrocenyl 3,4-Dimethoxybenzoate (1)}

Yield $76 \%$, yellow prisms from methanol. Mp.: $131^{\circ} \mathrm{C}-134^{\circ} \mathrm{C}$. FT-IR (Nujol, $\mathrm{cm}^{-1}$ ): 3078, 1727, 1596, 1517 , 1417, 1349, 1289, 1273, 1250, 1233, 1214, 1193, 1172, 1142, 1105, 1084, 1025, 1001, 931, 905, 893, 810, 768, 758, 609, 513, 501, 489. ${ }^{1} \mathrm{H}$ NMR $\left(300 \mathrm{MHz}, \mathrm{CDCl}_{3}\right): \delta 3.94(3 \mathrm{H}, \mathrm{s}, \mathrm{MeO}), 3.95(3 \mathrm{H}, \mathrm{s}, \mathrm{MeO}), 4.00(2 \mathrm{H}, \mathrm{s}, \mathrm{Fc})$, $4.26(5 \mathrm{H}, \mathrm{s}, \mathrm{Fc}), 4.56(2 \mathrm{H}, \mathrm{s}, \mathrm{Fc}), 6.76\left(1 \mathrm{H}, \mathrm{d}, J=8.4 \mathrm{~Hz}, \mathrm{H}-5^{\prime}\right), 7.58\left(1 \mathrm{H}, \mathrm{s}, \mathrm{H}-2^{\prime}\right), 7.75\left(1 \mathrm{H}, \mathrm{d}, J=8.4 \mathrm{~Hz}, \mathrm{H}-6^{\prime}\right)$. ${ }^{13} \mathrm{C} \mathrm{NHC}\left(75 \mathrm{MHz}, \mathrm{CDCl}_{3}\right): \delta 55.99,60.87,63.28,69.35,110.23,112.08,116.23,122.05,123.93,148.64$, 153.25, 164.64. EI-MS (rel. int.\%): 367 (5) $[\mathrm{M}+1]^{+}, 336$ (24) [M] $]^{+}$. Anal. Calc. for $\mathrm{C}_{16} \mathrm{H}_{13} \mathrm{FeNO}_{2}: \mathrm{C}, 62.3 ; \mathrm{H}$, 4.9\%. Found: C, $62.0 ; \mathrm{H}, 4.9 \%$.

\subsection{Ferrocenyl 3,4,5-Trimethoxybenzoate (2)}

Yield $16 \%$, brown solid. Mp.: $123^{\circ} \mathrm{C}-127^{\circ} \mathrm{C}$. FT-IR (Nujol, $\mathrm{cm}^{-1}$ ): 1731, 1587, 1503, 1331, 1234, 1212, 1181, $1170,1130,1104,1097,1033,994,940,865,824,803,779,757,647,512,499 .{ }^{1} \mathrm{H}$ NMR $\left(300 \mathrm{MHz}, \mathrm{CDCl}_{3}\right): \delta$ $3.91(3 \mathrm{H}, \mathrm{s}, 4-\mathrm{MeO}), 3.93(6 \mathrm{H}, \mathrm{s}, 3,5-\mathrm{MeO}), 4.10(2 \mathrm{H}, \mathrm{s}, \mathrm{Fc}), 4.35(5 \mathrm{H}, \mathrm{s}, \mathrm{Fc}), 4.67(2 \mathrm{H}, \mathrm{s}, \mathrm{Fc}), 7.33(2 \mathrm{H}, \mathrm{s}$, Ar-2,6). ${ }^{13} \mathrm{C}$ NMR (75 MHz, $\left.\mathrm{CDCl}_{3}\right): \delta 45.42,56.34,60.94,61.27,63.99,70.25,107.16,152.95,161.38,164.32$. EI-MS (rel. int.\%): 397 (8) [M+1] $]^{+}, 396(25)[\mathrm{M}]^{+}$. Anal. Calc. for $\mathrm{C}_{20} \mathrm{H}_{20} \mathrm{FeO}_{5}: \mathrm{C}, 60.6 ; \mathrm{H}$, 5.1. Found: C, 60.7; $\mathrm{H}, 5.5 \%$. 


\subsection{Ferrocenyl 4-(Trifluromethyl)-Benzoate (3)}

Yield 35\%, red powder, Mp.: $106^{\circ} \mathrm{C}-107^{\circ} \mathrm{C}$. FT-IR (Nujol, $\mathrm{cm}^{-1}$ ): 3117, 1735, 1695, 1412, 1334, 1307, 1281, $1238,1161,1115,1107,1095,1069,1019,1001,927,859,814,768,698,662,592,513,484 .{ }^{1} \mathrm{H}$ NMR (300 $\left.\mathrm{MHz}, \mathrm{CDCl}_{3}\right): \delta 4.01(2 \mathrm{H}, \mathrm{t}, J=1.8 \mathrm{~Hz}, \mathrm{Fc}), 4.25(5 \mathrm{H}, \mathrm{s}, \mathrm{Fc}), 4.57(2 \mathrm{H}, \mathrm{t}, J=1.8 \mathrm{~Hz}, \mathrm{Fc}), 7.74(2 \mathrm{H}, \mathrm{d}, J=8.1$ $\left.\mathrm{Hz}, \mathrm{H}-3^{\prime}, 5^{\prime}\right), 8.72\left(2 \mathrm{H}, \mathrm{d}, J=8.1 \mathrm{~Hz}, \mathrm{H}-2{ }^{\prime}, 6{ }^{\prime}\right) .{ }^{13} \mathrm{C} \mathrm{NMR}\left(75 \mathrm{MHz}, \mathrm{CDCl}_{3}\right): \delta 60.84,63.46,69.42,99,95$, 125.45, 125.55, 130.22. EI-MS (rel. int.\%): 375 (12) $[\mathrm{M}+1]^{+}, 374$ (49) $[\mathrm{M}]^{+}$. Anal. Calc. for $\mathrm{C}_{18} \mathrm{H}_{13} \mathrm{FeO}_{2}: \mathrm{C}$, 57.78; H, 3.50. Found: C 57.76; H 3.49\%.

\subsection{Ferrocenyl Naphthalene-1-Carboxylate (4)}

Yield $76 \%$, red crystals, Mp.: $95^{\circ} \mathrm{C}-101^{\circ} \mathrm{C}$. FT-IR (Nujol, $\mathrm{cm}^{-1}$ ): 1732, 1690, 1593, 1575, 1510, 1411, 1348, $1276,1231,1189,1121,1106,1069,1032,1024,1001,987,923,869,810,779,648,610,559,509,489 .{ }^{1} \mathrm{H}$ NMR (300 MHz, CDCl $): \delta 4.03(2 \mathrm{H}, \mathrm{t}, J=1.8 \mathrm{~Hz}, \mathrm{Fc}), 4.29(5 \mathrm{H}, \mathrm{s}, \mathrm{Fc}), 4.62(2 \mathrm{H}, J=2.0 \mathrm{~Hz}, \mathrm{Fc}), 7.50-7.70$ $\left(3 \mathrm{H}, \mathrm{m}, \mathrm{H}-7^{\prime}, 6^{\prime}, 3^{\prime}\right), 7.89\left(1 \mathrm{H}, \mathrm{d}, J=8.1 \mathrm{~Hz}, \mathrm{H}-5^{\prime}\right), 8.05\left(1 \mathrm{H}, \mathrm{d}, J=8.1 \mathrm{~Hz}, \mathrm{H}-4^{\prime}\right), 8.28(1 \mathrm{H}, \mathrm{dd}, J=7.4 \mathrm{~Hz}, J=$ $\left.1.1 \mathrm{~Hz}, \mathrm{H}-2^{\prime}\right), 8.96\left(1 \mathrm{H}, \mathrm{d}, J=8.7 \mathrm{~Hz}, \mathrm{H}-8^{\prime}\right) .{ }^{13} \mathrm{C} \mathrm{NMR}\left(75 \mathrm{MHz}, \mathrm{CDCl}_{3}\right): \delta 61.13,63.42,69.39,124.49,125.65$, 126.34, 128.01, 128.62, 130.66, 133.86, 133.87. EI-MS (rel. int.\%): 357 (12) $[\mathrm{M}+1]^{+}, 356$ (48) [M] $]^{+}$. Anal. Calc. for $\mathrm{C}_{18} \mathrm{H}_{13} \mathrm{FeO}_{2}$ : C 70.81; H 4.53. Found: C, 70.61; H, 4.20\%.

\subsection{Ferrocenyl Cinnamate (5)}

Yield $36 \%$, yellow crystals. Mp.: $82^{\circ} \mathrm{C}-83.5^{\circ} \mathrm{C}$. (lit.: $88.5^{\circ} \mathrm{C}-90^{\circ} \mathrm{C}$ [44]). FT-IR (Nujol, $\mathrm{cm}^{-1}$ ): 3108,1732 , $1642,1331,1310,1234,1157,1105,979,804,764,516,491 .{ }^{1} \mathrm{H}$ NMR $\left(300 \mathrm{MHz}, \mathrm{CDCl}_{3}\right): \delta 4.17(2 \mathrm{H}, \mathrm{s}, \mathrm{Fc})$, $4.43(5 \mathrm{H}, \mathrm{s}, \mathrm{Fc}), 4.70(2 \mathrm{H}, \mathrm{s}, \mathrm{Fc}), 6.46(1 \mathrm{H}, \mathrm{d}, J=15.6 \mathrm{~Hz}, \mathrm{CO}-\mathrm{CH}=), 7.40\left(3 \mathrm{H}, \mathrm{m}, \mathrm{H}-2^{\prime}, 4^{\prime}, 6^{\prime}\right), 7.54(2 \mathrm{H}, \mathrm{m}$, H-3',5'), $7.71(1 \mathrm{H}, \mathrm{d}, J=15.6 \mathrm{~Hz}, \mathrm{Ar}-\mathrm{CH}=)$. EI-MS (rel. int.\%): $332(18)[\mathrm{M}]^{+}$.

\subsection{Ferrocenyl Furan-2-Carboxylate (6)}

Yield 34\%, orange powder, Mp.: $94^{\circ} \mathrm{C}-95^{\circ} \mathrm{C}$. FT-IR (Nujol, $\mathrm{cm}^{-1}$ ): 1741, 1578, 1568, 1552, 1410, 1393, 1293 , 1236, 1173, 1105, 1097, 1074, 1013, 935, 917, 884, 826, 809, 595, 492. ${ }^{1} \mathrm{H}$ NMR $\left(300 \mathrm{MHz}, \mathrm{CDCl}_{3}\right): \delta 4.01$ $(2 \mathrm{H}, \mathrm{t}, J=2.0 \mathrm{~Hz}, \mathrm{Fc}), 4.28(5 \mathrm{H}, \mathrm{s}, \mathrm{Fc}), 4.60(2 \mathrm{H}, \mathrm{t}, J=2.0 \mathrm{~Hz}, \mathrm{Fc}), 6.58(1 \mathrm{H}, \mathrm{dd}, J=3.5 \mathrm{~Hz}, J=1.7$ Гц, Н-4'), $7.30\left(1 \mathrm{H}, \mathrm{dd}, J=3.5 \mathrm{~Hz}, J=0.8 \mathrm{~Hz}, \mathrm{H}-3^{\prime}\right) 7.66\left(1 \mathrm{H}, \mathrm{dd}, J=1.7 \mathrm{~Hz}, J=0.8 \mathrm{~Hz}, \mathrm{H}-5^{\prime}\right) .{ }^{13} \mathrm{C}$ NMR $(75 \mathrm{MHz}$, $\left.\mathrm{CDCl}_{3}\right): \delta 60.26,62.78,68.95,111.51,115.46,118.19,143.76,146.28,156.14$. EI-MS (rel. int.\%): 297 (19) $[\mathrm{M}+1]^{+}, 296(100)[\mathrm{M}]^{+}$. Anal. Calc. for $\mathrm{C}_{15} \mathrm{H}_{12} \mathrm{FeO}_{3}$ : C, 60.84; H, 4.08. Found: C, 60.06; H, 4.00\%.

\subsection{Ferrocenyl Thiophene-2-Carboxylate (7)}

Yield 35\%, yellow solid. Mp.: $108^{\circ} \mathrm{C}-109^{\circ} \mathrm{C}$. FT-IR (Nujol, $\mathrm{cm}^{-1}$ ): 1734, 1522, 1355, 1266, 1245, 1233, 1213 , $1103,1076,1061,1018,1009,924,859,849,838,827,807,742,614,499,486 .{ }^{1} \mathrm{H}$ NMR $\left(300 \mathrm{MHz}, \mathrm{CDCl}_{3}\right): \delta$ $4.04(2 \mathrm{H}, \mathrm{s}, \mathrm{Fc}), 4.31(5 \mathrm{H}, \mathrm{s}, \mathrm{Fc}), 4.61(2 \mathrm{H}, \mathrm{s}, \mathrm{Fc}), 7.13(1 \mathrm{H}, \mathrm{dd}, J=4.8 \mathrm{~Hz}, J=2.7 \mathrm{~Hz}, \mathrm{H}-4), 7.60(1 \mathrm{H}, \mathrm{d}, J=$ $\left.4.8 \mathrm{~Hz}, \mathrm{H}-3^{\prime}\right), 7.86\left(1 \mathrm{H}, \mathrm{d}, J=2.7 \mathrm{~Hz}, \mathrm{H}-5^{\prime}\right) .{ }^{13} \mathrm{C}$ NMR $\left(75 \mathrm{MHz}, \mathrm{CDCl}_{3}\right): \delta 61.21,63.81,69.97,127.89,133.05$, 134.04, 160.25. EI-MS (rel. int.\%): $314(6)[\mathrm{M}+2]^{+}, 313(18)[\mathrm{M}+1]^{+}, 312(87)[\mathrm{M}]^{+}$. Anal. Calc. for $\mathrm{C}_{16} \mathrm{H}_{13} \mathrm{FeNO}_{2}$ : C, 57.7; H, 3.9; $\mathrm{S}, 10.3 \%$. Found: $\mathrm{C}, 57.5 ; \mathrm{H}, 4.0 ; \mathrm{S}, 10.1 \%$.

\subsection{Ferrocenyl Pyridine-2-Carboxylate (8)}

Yield 17\%, black solid. Compound is unstable in air (decomposes in $2-3$ days at room temperature). FT-IR (Nujol, $\mathrm{cm}^{-1}$ ): 3231, 1777, 1705, 1666, 1625, 1593, 1567, 1259, 1047, 858, 765, 663. ${ }^{1} \mathrm{H}$ NMR (300 MHz, $\left.\mathrm{CDCl}_{3}\right): \delta 4.02(2 \mathrm{H}, \mathrm{s}, \mathrm{Fc}), 4.26(5 \mathrm{H}, \mathrm{s}, \mathrm{Fc}), 4.66(2 \mathrm{H}, \mathrm{s}, \mathrm{Fc}), 7.53\left(1 \mathrm{H}, \mathrm{m}, \mathrm{H}-5^{\prime}\right), 7.89\left(1 \mathrm{H}, \mathrm{m}, \mathrm{H}-3^{\prime}\right), 8.19(1 \mathrm{H}$, m, H-4'), $8.82\left(1 \mathrm{H}, \mathrm{m}, \mathrm{H}-6^{\prime}\right) . \mathrm{NMR}{ }^{13} \mathrm{C}, \delta 60.32,62.85,69.01,124.94,126.59,126.60,136.59,140.87,149.64$, 162.74. EI-MS (rel. int.\%): $308(20)[\mathrm{M}+1]^{+}, 307(100)[\mathrm{M}]^{+} . \mathrm{C}_{16} \mathrm{H}_{13} \mathrm{FeNO}_{2}$. No correct elemental analysis could be obtained for this compound, despite several attempts.

\subsection{Ferrocenyl Pyridine-3-Carboxylate (9)}

Yield 33\%, brown solid. Compound is unstable in air. Mp.: $69^{\circ} \mathrm{C}-76^{\circ} \mathrm{C}$. FT-IR (Nujol, $\mathrm{cm}^{-1}$ ): 1730, 1679, 
$1279,1235,1103,1086,1038,1027,835,809,700,516,506,492 .{ }^{1} \mathrm{H}$ NMR $\left(300 \mathrm{MHz}, \mathrm{CDCl}_{3}\right): \delta 4.03(2 \mathrm{H}, \mathrm{s}$, Fc), 4.27 (5H, s, Fc), 4.58 (2H, s, Fc), 7.48 (1H, m, H-5'), 8.37 (1H, d, J = 6.9 Hz, H-4'), 8.88 (1H, m, H-6'), $9.36\left(1 \mathrm{H}, \mathrm{s}, \mathrm{H}-2^{\prime}\right) . \mathrm{NMR}{ }^{13} \mathrm{C}\left(75 \mathrm{MHz}, \mathrm{CDCl}_{3}\right): \delta 60.42,63.01,68.98,115.52,123.12,129.21$ 136.77, 150.69, 153.26, 163.18. EI-MS (rel. int.\%): 308 (17) $[\mathrm{M}+1]^{+}, 307(86)[\mathrm{M}]^{+} . \mathrm{C}_{16} \mathrm{H}_{13} \mathrm{FeNO}_{2}$. We failed to obtain correct elemental analysis for this compound.

\subsection{Ferrocenyl Pyridine-4-Carboxylate (10)}

Yield 17\%, brown prisms from hexanes-AcOEt. Mp.: $82^{\circ} \mathrm{C}-85^{\circ} \mathrm{C}$. FT-IR (Nujol, cm ${ }^{-1}$ ): $1748,1712,1675,1351$, $1324,1272,1234,1104,1064,923,818,753,701,490 .{ }^{1} \mathrm{H}$ NMR $\left(300 \mathrm{MHz}, \mathrm{CDCl}_{3}\right): \delta 4.03(2 \mathrm{H}, \mathrm{s}, \mathrm{Fc}), 4.26$ $(5 \mathrm{H}, \mathrm{s}, \mathrm{Fc}), 4.58(2 \mathrm{H}, \mathrm{s}, \mathrm{Fc}), 7.93\left(2 \mathrm{H}, \mathrm{m}, \mathrm{H}-3^{\prime}, 5^{\prime}\right), 8.87\left(2 \mathrm{H}, \mathrm{m}, \mathrm{H}-2^{\prime}, 66^{\prime}\right) .{ }^{13} \mathrm{C} \mathrm{NHC}\left(75 \mathrm{MHz}, \mathrm{CDCl}_{3}\right): \delta 60.74$, 63.48, 69.42, 122.90, 129.38, 129.61, 150.71, 163.42. EI-MS (rel. int.\%): 308 (21) [M+1] $]^{+}, 307$ (100) [M]. Anal. Calc. for $\mathrm{C}_{16} \mathrm{H}_{13} \mathrm{FeNO}_{2}$ : C, 62.6; H, 4.3; N, 4.6\%. Found: C, 62.9; H, 4.7; N, 3.9\%.

\subsection{Ferrocenyl Ferrocenate (11)}

This compound is already known [41]. Yield 28\%, brown crystals from methanol. Mp.: $148^{\circ} \mathrm{C}-149^{\circ} \mathrm{C}$. FT-IR (Nujol, $\mathrm{cm}^{-1}$ ): 1717, 1411, 1351, 1274, 1230, 1114, 1105, 1055, 1047, 1035, 1018, 1003, 927, 836, 827, 814, 761, 533, 501, 487, 463. ${ }^{1} \mathrm{H} \mathrm{NMR}\left(300 \mathrm{MHz}, \mathrm{CDCl}_{3}\right): \delta 3.98(2 \mathrm{H}, \mathrm{t}, J=2.0 \mathrm{~Hz}, \mathrm{Fc}), 4.25(5 \mathrm{H}, \mathrm{s}, \mathrm{Fc}), 4.26(5 \mathrm{H}$, $\mathrm{s}, \mathrm{Fc}), 4.45(2 \mathrm{H}, \mathrm{t}, J=2.0 \mathrm{~Hz}, \mathrm{Fc}), 4.51(2 \mathrm{H}, \mathrm{t}, J=2.0 \mathrm{~Hz}, \mathrm{Fc}), 4.88(2 \mathrm{H}, \mathrm{t}, J=2.0 \mathrm{~Hz}, \mathrm{Fc}) .{ }^{13} \mathrm{C} \mathrm{NMR}(75 \mathrm{MHz}$ $\mathrm{CDCl}_{3}$ ): $\delta 60.79,63.15,69.30,69.88,70.38,71.70$. EI-MS (rel. int.\%): $415(18)[\mathrm{M}+1]^{+}, 414(55)[\mathrm{M}]^{+}$. Anal. Calc. for $\mathrm{C}_{21} \mathrm{H}_{18} \mathrm{Fe}_{2} \mathrm{O}_{2}$ : C, 60.9; H, 4.4. Found: C, 60.1; H, 4.6\%.

\subsection{Crystal Structure Determination}

The unit cell parameters and the X-ray diffraction intensities of compounds $\mathbf{1}, \mathbf{4}$ were measured on a Xcalibur R diffractometer. The empirical absorption correction was introduced by multi-scan method using SCALE3 ABSPACK algorithm [56]. The structures were solved by direct method and refined by the full-matrix leastsquares method in the anisotropic approximation for all non-hydrogen atoms using the SHELX-97 program package [57]. Hydrogen atoms were located from the Fourier synthesis of the electron density and refined using a riding model.

A suitable crystal of compound $\mathbf{1 0}$ was selected and XRD analysis was accomplished on a Xcalibur, Eos diffractometer on standard procedure (MoK-irradiation, graphite monochromator, $\mathrm{T}=295(2) \mathrm{K}$, $\omega$-scanning with $1^{\circ}$ step). Empirical absorption correction was applied [58]. Using Olex2 [59], the structure was solved with the Superflip [60] structure solution program using Charge Flipping and refined with the ShelXL [57] refinement package using Least Squares minimization.

Crystal Data of 1. $\mathrm{C}_{19} \mathrm{H}_{18} \mathrm{FeO}_{4}, M=366.18$, triclinic, $a=7.8682(9) \AA, b=10.2613(10) \AA, c=11.2587(13) \AA$, $\alpha=110.514(10)^{\circ}, \beta=104.022(10)^{\circ}, \gamma=104.022(10)^{\circ}, V=816.95(15) \AA^{3}, T=295(2)$, space group P-1, $Z=2, \mu$ $(\mathrm{Mo} \mathrm{K} \alpha)=0.942 \mathrm{~mm}^{-1}$. The final refinement parameters: $R_{1}=0.0407, w R_{2}=0.0924$ (for all independent 3766 reflections, $R_{\text {int }}=0.0259$ ); $R_{1}=0.0349, w R_{2}=0.0879$ [for observed 3347 reflections with $I>2 \sigma(I)$ ], $S=1.059$. Largest diff. peak and hole 0.256 and $-0.421 \overline{\mathrm{e}} \AA^{-3}$.

Crystal Data of 4. $\mathrm{C}_{21} \mathrm{H}_{16} \mathrm{FeO}_{2}, M=356.19$, monoclinic, $a=12.2242(18) \AA, b=7.6261(13) \AA, c=17.941(3)$

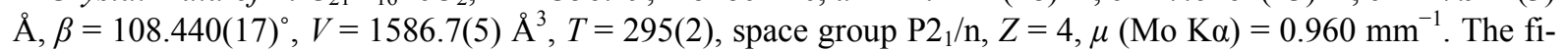
nal refinement parameters: $R_{1}=0.0457, w R_{2}=0.1043$ (for all independent 3686 reflections, $R_{\text {int }}=0.0347$ ); $R_{1}=$ $0.0385, w R_{2}=0.0999$ [for observed 3165 reflections with $I>2 \sigma(I)$ ], $S=1.064$. Largest diff. peak and hole 0.427 and $-0.473 \overline{\mathrm{e}} \AA^{-3}$.

Crystal Data of 10. $\mathrm{C}_{16} \mathrm{H}_{13} \mathrm{FeNO}_{2}, M=306.13$, triclinic, $a=7.5025(2) \AA, b=10.7990(4) \AA, c=16.9885(7) \AA$, $\alpha=98.444(3)^{\circ}, \beta=100.017(3)^{\circ}, \gamma=90.630(3)^{\circ}, V=1339.78(9) \AA^{3}, T=295(2)$, space group P-1, $Z=4, \mu(\mathrm{Mo}$ $\mathrm{K} \alpha)=1.125 \mathrm{~mm}^{-1}$. On the angles $1.91<\theta<30.80^{\circ} 12392$ reflections measured, 7233 unique (5201 with $\mathrm{I}>$ $\left.2 \sigma(\mathrm{I}), R_{\text {int }}=0.0287\right)$ which were used in all calculations. Completeness for $\theta<26.0^{\circ} 100 \%$. The final $w R_{2}$ was 0.1495 (all data) and $R_{1}$ was $0.0426(\mathrm{I}>2 \sigma(\mathrm{I}))$. Largest diff. peak and hole 0.564 and $-0.431 \overline{\mathrm{e}} \AA^{-3}$.

\section{Conclusion}

Herein, we disclose the simple straightforward method for synthesis of ferrocenol esters, beginning with easily 
accessible ferrocenol boronic acid and aryl-(hetaryl)-aldehydes. The reaction is catalyzed by N-heterocyclic carbene (IMes) and is suitable for synthesis of substituted benzoic acid, as well as for heterocyclic acids; however, 2- and 3-pyridylcarboxylic acid ferrocenol esters are rather unstable. Limitation and scope of the method are currently under investigation in our laboratory.

\section{Acknowledgements}

The authors thank engineer I.A. Borisova for recording the IR spectra, leading engineer O.A. Maiorova for recording the ${ }^{1} \mathrm{H}$ and ${ }^{13} \mathrm{C}$ NMR spectra, and researcher E.V. Baigacheva for performing elemental analyses. This study was performed under financial support by the Russian Foundation for Basic Research (Projects No. 14-03-31168-mol-a, 16-33-00147-mol-a).

\section{Supplementary Material}

CCDC 1034705 (compound 10), 1453993 (compound 1) and 1453994 (compound 4) contain the supplementary crystallographic data for this paper. The data can be obtained free of charge from The Cambridge Crystallographic Data Centre via http://www.ccdc.cam.ac.uk.

\section{References}

[1] Hayashi, T. and Togni, A. (1995) Ferrocenes. VCH, Weinheim.

[2] Štěpnička, P. (2008) Ferrocenes: Ligands, Materials, Biomolecules. John Wiley \& Sons, Chichester.

[3] Phillips, E.S. (2011) Ferrocenes: Compounds, Properties, and Applications. Nova Science Publishers, Hauppauge.

[4] Braga, S.S. and Silva, A.M.S. (2013) A New Age for Iron: Antitumoral Ferrocenes. Organometallics, 32, 5626-5639. http://dx.doi.org/10.1021/om400446y

[5] Orlenas, C. (2011) Application of Ferrocene and Its Derivatives in Cancer Research. New Journal of Chemistry, 35, 1973-1985. http://dx.doi.org/10.1039/clnj20172g

[6] Snegur, L.V., Babin, V.N., Simenel, A.A., Nekrasov, Yu.S., Ostrovskaya, L.A. and Sergeeva, N.S. (2010) Antitumor Activities of Ferrocene Compounds. Russian Chemical Bulletin, 59, 2167-2178.

[7] Manosroi, J., Rueanto, K., Boonpisuttinant, K., Manosroi, W., Biot, C., Akazawa, H., Akihisa, T., Issarangporn, W. and Manosroi, A. (2010) Novel Ferrocenic Steroidal Drug Derivatives and Their Bioactivities. Journal of Medicinal Chemistry, 53, 3937-3943. http://dx.doi.org/10.1021/jm901866m

[8] Top, S., Vessiéres, A., Leclercq, G., Quivy, J., Tang, J., Vaissermann, J., Huché, M. and Jaouen, G. (2003) Synthesis, Biochemical Properties and Molecular Modelling Studies of Organometallic Specific Estrogen Receptor Modulators (SERMs), the Ferrocifens and Hydroxyferrocifens: Evidence for an Antiproliferative Effect of Hydroxyferrocifens on both Hormone-Dependent and Hormone-Independent Breast Cancer Cell Lines. Chemistry: A European Journal, 9 , 5223-5236. http://dx.doi.org/10.1002/chem.200305024

[9] Top, S., Dauer, B., Vaissermann, J. and Jaouen, G. (1997) Facile Route to Ferrocifen, 1-[4-(2-Dimethylaminoethoxy)]-(Phenyl-2-Ferrocenyl-But-1-Ene), First Organometallic Analogue of Tamoxifen, by the McMurry Reaction. Journal of Organometallic Chemistry, 541, 355-361. http://dx.doi.org/10.1016/S0022-328X(97)00086-7

[10] Jios, J.L., Kirin, S.I., Buceta, N.N., Weyhermüller, T., Della Védova, C.O. and Metzler-Nolte, N. (2007) Synthesis and Structural Characterization of Metallated Bioconjugates: C-Terminal Labeling of Amino Acids with Aminoferrocene. Journal of Organometallic Chemistry, 692, 4209-4214. http://dx.doi.org/10.1016/j.jorganchem.2007.06.044

[11] Otón, F., Espinosa, A., Tárraga, A., de Arellano, C.R. and Molina, P. (2007) Ferrocenophanes with Guanidine Bridging Units as Multisignalling Receptor Molecules for Selective Recognition of Anions, Cations, and Amino Acids. Chemistry: A European Journal, 13, 5742-5752. http://dx.doi.org/10.1002/chem.200601757

[12] Görmen, M., Pigeon, P., Top, S., Hillard, E.A., Huché, M., Hartinger, C.G., de Montigny, F., Plamont, M.-A., Vessières, A. and Jaouen, G. (2010) Synthesis, Cytotoxicity, and COMPARE Analysis of Ferrocene and Ferrocenophane Tetrasubstituted Olefin Derivatives against Human Cancer Cells. ChemMedChem, 5, 2039-2050. http://dx.doi.org/10.1002/cmdc.201000286

[13] Abri, M.E., Pigeon, P., Top, S., Rhouma, A., Aifa, S., Rebai, A., Vessières, A., Plamont, M.-A. and Jaouen, G. (2011) Evaluation of Bactericidal and Fungicidal Activity of Ferrocenyl or Phenyl Derivatives in the Diphenyl Butene Series. Journal of Organometallic Chemistry, 696, 1038-1048. http://dx.doi.org/10.1016/j.jorganchem.2010.09.015

[14] Harry, A.G., Butler, W.E., Manton, J.C., Pryce, M.T., O’Donovan, N., Crown, J., Rai, D.K. and Kenny, P.T.M. (2014) The Synthesis, Structural Characterization and in Vitro Anti-Cancer Activity of Novel 1-Alkyl-1'-N-Para-(Ferrocenyl) 
Benzoyl Dipeptide Esters. Journal of Organometallic Chemistry, 757, 28-35. http://dx.doi.org/10.1016/j.jorganchem.2014.01.031

[15] Hottin, A., Dubar, F., Steenackers, A., Delannoy, P., Biot, C. and Behr, J.-B. (2012) Iminosugar-Ferrocene Conjugates as Potential Anticancer Agents. Organic \& Biomolecular Chemistry, 10, 5592-5597. http://dx.doi.org/10.1039/c2ob25727k

[16] Huber, D., Hubner, H. and Gmeiner, P. (2009) 1,1'-Disubstituted Ferrocenes as Molecular Hinges in Mono- and Bivalent Dopamine Receptor Ligands. Journal of Medicinal Chemistry, 52, 6860-6870. http://dx.doi.org/10.1021/jm901120h

[17] Braga, D., Giaffreda, S.L., Grepioni, F., Palladino, G. and Polito, M. (2008) Mechanochemical Assembly of Hybrid Organic-Organometallic Materials. Solid-Solid Reactions of 1,1'-di-Pyridyl-Ferrocene with Organic Acids. New Journal of Chemistry, 32, 820-828. http://dx.doi.org/10.1039/b714923a

[18] Zhu, Y. and Wolf, M.O. (2000) Charge Transfer and Delocalization in Conjugated (Ferrocenylethynyl)oligothiophene Complexes. Journal of the American Chemical Society, 122, 10121-10125. http://dx.doi.org/10.1021/ja0008564

[19] Tonhauser, C., Alkan, A., Schömer, A.M., Dingels, C., Ritz, S., Mailänder, V., Frey, H. and Wurm, F.R. (2013) Ferrocenyl Glycidyl Ether: A Versatile Ferrocene Monomer for Copolymerization with Ethylene Oxide to Water-Soluble, Thermoresponsive Copolymers. Macromolecules, 46, 647-655. http://dx.doi.org/10.1021/ma302241w

[20] Schacher, F.H., Rupar, P.A. and Manners, I. (2012) Functional Block Copolymers: Nanostructured Materials with Emerging Applications. Angewandte Chemie International Edition, 51, 7898-7921. http://dx.doi.org/10.1002/anie.201200310

[21] Antufjeva, A.D., Kabelitskaya, Y.M., Shklyaeva, E.V. and Abashev, G.G. (2013) Ferrocene-Containing Compounds for Material Chemistry. I. Ferrocene-Containing Polymers. Vestnik Permskogo Yuniversiteta: Chemistry, 4, 59-81.

[22] Dai, L.-X. and Hou, X.-L. (2010) Chiral Ferrocenes in Asymmetric Catalysis: Synthesis and Applications. Wiley$\mathrm{VCH}$, Weinheim.

[23] Dai, L.-X., Tu, T., You, S.-L., Deng, W.-P. and Hou, X.-L. (2003) Asymmetric Catalysis with Chiral Ferrocene Ligands. Accounts of Chemical Research, 36, 659-667. http://dx.doi.org/10.1021/ar020153m

[24] Mamane, V. (2010) The Diastereoselective ortho-Lithiation of Kagan's Ferrocenyl Acetal. Generation and Reactivity of Chiral 2-Substituted Ferrocenecarboxaldehydes. Tetrahedron: Asymmetry, 21, 1019-1029. http://dx.doi.org/10.1016/j.tetasy.2010.03.012

[25] Chupakhin, O.N., Utepova, I.A., Kovalev, I.S., Rusinov, V.L. and Starikova, Z.A. (2007) Direct C-C Coupling of Ferrocenyllithium and Azaheterocycles by Nucleophilic Substitution of Hydrogen-Synthesis of Mono- and 1,1'-Diazinylferrocenes. European Journal of Organic Chemistry, 2007, 857-862. http://dx.doi.org/10.1002/ejoc.200600821

[26] Molina, P., Tárraga, A. and Alfonso, M. (2014) Ferrocene-Based Multichannel Ion-Pair Recognition Receptors. Dalton Transactions, 43, 18-29. http://dx.doi.org/10.1039/C3DT52459K

[27] Nicot, B.D., Maynadié, J., Lavabre, D. and Fery-Forgues, S.J. (2007) $\mathrm{Ca}^{2+}$ vs. $\mathrm{Ba}^{2+}$ Electrochemical Detection by Two Disubstituted Ferrocenyl Chalcone Chemosensors. Study of the Ligand-Metal Interactions in $\mathrm{CH}_{3} \mathrm{CN}$. Journal of Organometallic Chemistry, 692, 874-886.

[28] Liu, S., Wollenberger, U., Katterle, M. and Scheller, F. (2006) Ferroceneboronic Acid-Based Amperometric Biosensor for Glycated Hemoglobin. Sensors and Actuators B, 113, 623-629. http://dx.doi.org/10.1016/j.snb.2005.07.011

[29] Palomera, N., Vera, J.L., Meléndez, E., Ramirez-Vick, J.E., Tomar, M.S., Arya, S.K. and Singh, S.P. (2011) Redox Active Poly(Pyrrole-N-Ferrocene-Pyrrole) Copolymer Based Mediator-Less Biosensors. Journal of Electroanalytical Chemistry, 658, 33-37. http://dx.doi.org/10.1016/j.jelechem.2011.04.019

[30] Loubster, C. and Imrie, C. (1997) Thermal Properties of Monosubstituted Ferrocene Derivatives: A Series of New Ferrocenomesogens. Journal of the Chemical Society, Perkin Transactions 2, 399-410. http://dx.doi.org/10.1039/a600739b

[31] Imrie, C. and Loubster, C. (1994) A Liquid Crystalline Ferrocene Derivative with a Chiral Smectic c Phase. Journal of the Chemical Society, Chemical Communications, 2159-2160. http://dx.doi.org/10.1039/c39940002159

[32] Hildebrandt, A. and Lang, H. (2013) (Multi)ferrocenyl Five-Membered Heterocycles: Excellent Connecting Units for Electron Transfer Studies. Organometallics, 32, 5640-5653. http://dx.doi.org/10.1021/om400453m

[33] Edinç, P., Önal, A.M. and Özkar, S. (2007) Synthesis, Characterization, and Electrochemistry of Tetracarbonyl(6-Ferrocenyl-2,2'-bipyridine)tungsten(0). Journal of Organometallic Chemistry, 692, 1983-1989. http://dx.doi.org/10.1016/j.jorganchem.2007.01.006

[34] Rajput, J., Hutton, A.T., Moss, J.R., Su, H. and Imrie, C. (2006) Ferrocenyl-Nitrogen Donor Ligands. Synthesis and Characterization of Rhodium (I) Complexes of Ferrocenylpyridine and Related Ligands. Journal of Organometallic Chemistry, 691, 4573-4588. 
[35] Daeneke, T., Kwon, T.-H., Holmes, A., Duffy, N., Bach, U. and Spiccia, L. (2011) High-Efficiency Dye-Sensitized Solar Cells with Ferrocene-Based Electrolytes. Nature Chemistry, 3, 211-215. http://dx.doi.org/10.1038/nchem.966

[36] Nemykhin, V.N., Rohde, G.T., Barrett, C.D., Hadt, R.G., Sabin, J.R., Galloni, P. and Floris, B. (2010) Long-Range Electronic Communication in Free-Base meso-Poly(Ferrocenyl)-Containing Porphyrins. Inorganic Chemistry, 49, 7497-7509. http://dx.doi.org/10.1021/ic101012a

[37] Staff, R.F., Gallei, M., Mazureowski, M., Rehahn, M., Berger, R. and Landfester, K. (2012) Patchy Nanocapsules of Poly(Vinylferrocene)-Based Block Copolymers for Redox-Responsive Release. ACS Nano, 6, 9042-9049. http://dx.doi.org/10.1021/nn3031589

[38] Nesmeyanov, A.N., Sazonova, V.A. and Drozd, V.N. (1959) Oxyferrocene. Doklady Akademii Nauk SSSR, 129, 10601063.

[39] Nesmeyanov, A.N., Sazonova, V.A. and Drozd, V.N. (1959) Hydroxyferrocene. Tetrahedron Letters, 1, 13-15. http://dx.doi.org/10.1016/S0040-4039(00)91032-3

[40] Nesmeyanov, A.N., Sazonova, V.A., Drozd, V.N. and Nikonova, L.A. (1960) Oxyferroceny I ikh Proizvodnye. Doklady Akademii Nauk SSSR, 133, 126-129.

[41] Herberhold, M., Brendel, H.-D., Hofmann, A., Hofmann, B. and Milius, W. (1998) Neue Derivate des Ferrocenols und 1,1'-Ferrocendiols. Journal of Organometallic Chemistry, 556, 173-187. http://dx.doi.org/10.1016/S0022-328X(97)00716-X

[42] Sato, M., Motoyama, I. and Hata, K. (1970) Reactions of Haloferrocenes. III. The Reaction of Haloferrocenes with Copper(I) Benzenethiolate, Thiocyanate, Acetate, and Benzoate. Bulletin of the Chemical Society of Japan, 43, 22132217. http://dx.doi.org/10.1246/bcsj.43.2213

[43] Sato, M., Lam, Y.P., Motoyama, I. and Hata, K. (1971) Reactions of Haloferrocenes. IV. The Condensation of Haloferrocenes with Various Carboxylic Acids in the Presence of Copper (I) Oxide. Bulletin of the Chemical Society of Japan, 44, 808-812. http://dx.doi.org/10.1246/bcsj.44.808

[44] Akabori, S., Sato, M. and Ebine, S. (1981) Convenient Preparation of Ferrocenyl Esters and Ethers. Synthesis, 1981, 278-279. http://dx.doi.org/10.1055/s-1981-29413

[45] Epton, R., Marr, G. and Rogers, G.K. (1978) The Synthesis and Reactivity of (Ferrocenyloxy)-2-tetrahydropyran. Journal of Organometallic Chemistry, 150, 93-100.

[46] Floris, B. and Illuminati, G. (1978) The Kinetics and Mechanism of the Protodeboronation of Ferroceneboronic Acid in Moderately Concentrated Sulfuric Acid. Journal of Organometallic Chemistry, 150, 101-113. http://dx.doi.org/10.1016/S0022-328X(00)85553-9

[47] Barba, V., Xochipa, R., Santillan, R. and Farfan, N. (2004) Study of the Reaction of Tridentate Ligands with Ferrocenyl Boronic Acid. European Journal of Inorganic Chemistry, 2004, 118-124. http://dx.doi.org/10.1002/ejic.200300337

[48] Denisov, M.S., Antuf'eva, A.D., Gorbunov, A.A., Manylova, K.O. and Glushkov, V.A. (2015) Esters of Ferrocenol: Synthesis, Optical Properties, and Electrochemical Behavior. Russian Journal of General Chemistry, 85, 2765-2770. http://dx.doi.org/10.1134/S107036321512018X

[49] Sarkar, S.D., Biswas, A., Samanta, R.C. and Studer, A. (2013) Catalysis with $N$-Heterocyclic Carbenes under Oxidative Conditions. Chemistry - A European Journal, 19, 4664-4678. http://dx.doi.org/10.1002/chem.201203707

[50] Knappke, C.E.I., Imami, A. and von Wangelin, A.J. (2012) Oxidative $N$-Heterocyclic Carbene Catalysis. ChemCatChem, 4, 937-941. http://dx.doi.org/10.1002/cctc.201200133

[51] Nair, V., Menon, R.S., Biju, A.T., Sinu, C.R., Paul, R.R., Jose, A. and Sreekumar, V. (2011) Employing Homoenolates Generated by NHC Catalysis in Carbon-Carbon Bond-Forming Reactions: State of the Art. Chemical Society Reviews, 40, 5336-5346. http://dx.doi.org/10.1039/c1cs15139h

[52] Meng, J.-J., Gao, M., Wei, Y.-P. and Zhang, W.-Q. (2012) N-Heterocyclic Carbene-Catalyzed Aerobic Oxidative Direct Esterification of Aldehydes with Organoboronic Acids. Chemistry-An Asian Journal, 7, 872-875. http://dx.doi.org/10.1002/asia.201200076

[53] Arde, P., Ramanjaneyulu, B.T., Reddy, V., Saxena, A. and Anand, R.V. (2012) N-Heterocyclic Carbene Catalysed Aerobic Oxidation of Aromatic Aldehydes to Aryl Esters Using Boronic Acids. Organic \& Biomolecular Chemistry, 10, 848-851. http://dx.doi.org/10.1039/C1OB06566A

[54] Denisov, M.S., Gorbunov, A.A. and Glushkov, V.A. (2015) N-Heterocyclic Carbenes: IX. Oxidative Esterification of Aromatic Aldehydes with Arylboronic Acids Catalyzed by $N$-Heterocyclic Carbenes. Russian Journal of Organic Chemistry, 51, 86-90. http://dx.doi.org/10.1134/s1070428015010145

[55] Arduengo III, A.J., Krafczyk, R., Schmutzler, R., Craig, H.A., Goerlich, J.R., Marshall, W.J. and Unverzagt, M. (1999) Imidazolylidenes, Imidazolinylidenes and Imidazolidines. Tetrahedron, 55, 14523-15534. 
http://dx.doi.org/10.1016/S0040-4020(99)00927-8

[56] CrysAlisPro, Agilent Technologies. Version 1.171.37.33 (Release 27-03-2014 CrysAlis171 .NET).

[57] Sheldrick, G.M. (2008) A Short History of SHELX. Acta Crystallographica Section A, 64, 112-122.

[58] Dolomanov, O.V., Bourhis, L.J., Gildea, R.J., Howard, J.A.K. and Puschmann, H. (2009) OLEX2: A Complete Structure Solution, Refinement and Analysis Program. Journal of Applied Crystallography, 42, 339-341. http://dx.doi.org/10.1107/S0021889808042726

[59] Palatinus, L. and Chapuis, G. (2007) SUPERFLIP-A Computer Program for the Solution of Crystal Structures by Charge Flipping in Arbitrary Dimensions. Journal of Applied Crystallography, 40, 786-790. http://dx.doi.org/10.1107/S0021889807029238

[60] CrysAlisPro, Agilent Technologies. Version 1.171.36.28. 